\title{
Systematic Review: ClearCode 34 - A Validated Prognostic Signature in Clear Cell Renal Cell Carcinoma (ccRCC)
}

\author{
Pooja Ghatalia ${ }^{\mathrm{a}}$ and W. Kimryn Rathmell ${ }^{\mathrm{b}, *}$ \\ ${ }^{a}$ Department of Medical Oncology, Fox Chase Cancer Center, Philadelphia, PA, USA \\ ${ }^{\mathrm{b}}$ Department of Medicine, Division of Hematology and Oncology, Vanderbilt-Ingram Cancer Center, Nashville, \\ TN, USA
}

\begin{abstract}
.
Background: $\mathrm{ccA} / \mathrm{ccB}$ classification was developed to classify clear cell renal carcinoma (ccRCC) patients into high and low risk based on gene expression patterns. ClearCode 34 is a genetic signature that was developed from the $\mathrm{ccA} / \mathrm{ccB}$ classification to predict recurrence in localized ccRCC patients.

Objective: This review will evaluate the molecular signature ClearCode34, discuss its role in predicting recurrence and consider the rational application of the tool as a strategy to guide future applications of adjunctive therapy in ccRCC.

Methods: A review of all the relevant papers in PubMed with the terms "ccA/ccB" or "ClearCode34" in ccRCC were reviewed.

Results: Gene expression data was used to model dominant molecular subtypes of ccRCC tumors using consensus clustering methods. The most stable model implied two dominant subgroups - subsequently named ccA and ccB. A 34-gene panel was developed for clinical application, with 10 genes highly expressed corresponding to ccB subtype and 24 from ccA subtype. ClearCode34 independently correlated with cancer-specific survival, overall survival and recurrence in localized ccRCC patients in multiple validations.

Conclusions: ClearCode 34 is a robust and well validated molecular signature that can identify aggressive ccRCC in primary tumors. Along with basic clinical and pathologic variables like stage, necrosis and grade, robust molecular based prognostic markers are needed that could help better predict groups of patients who will most benefit from risk-adapted treatment approaches.
\end{abstract}

Keywords: Renal cell carcinoma, biomarker, prognostic, clearcode34, ccA, ccB

\section{INTRODUCTION}

Over 64,000 individuals will be diagnosed in the US annually with renal cell carcinoma. Fortunately, the great majority will have organ confined

${ }^{*}$ Correspondence to: W. Kimryn Rathmell, MD, PhD., Department of Medicine, Division of Hematology and Oncology, Vanderbilt-Ingram Cancer Center, 2220 Pierce Avenue, Preston Research Building, Suite 777, Vanderbilt University Medical Center, Nashville, TN 37232, USA. Tel.: +1 615875 9731; Fax: +1 615343 7602; E-mail: kimryn.rathmell@vanderbilt.edu. disease, although up to a third of these patients will go on to develop metastasis. Presently, the standard of care for patients who have received nephrectomy for localized clear cell renal cell carcinoma (ccRCC) is radiographic surveillance. Surveillance strategies are currently minimally stratified, and include a wide array of surveillance options, such that treating providers are limited in their ability to guide their patients using evidence-based guidelines in managing their risk for recurrence. 
The majority of risk stratification strategies for ccRCC have relied most heavily on clinical features to guide estimates of risk. Stage and grade remain the most widely used predictors of risk, given the exceptionally low risk of metastasis with T1a disease, and the exceptionally high risk with T3b/T4 stage. The Fuhrman grading system is similarly valuable for the rarely observed, but very indolent G1, and the highly aggressive G4. Additional adjectives can further define higher risk disease, such as the presence of sarcomatoid or rhabdoid histologic features. However, it is important to risk stratify patients with intermediate stage tumors (stage 2-3), whose clinical behavior may be hard to predict. Further, the distinctions between G2 and G3 are subject to user-dependent variation. Thus, the very patients for whom it is most challenging to estimate risk, are those for whom the clinical risk prediction algorithms are least equipped to provide objective and quantifiable information.

As options for adjunctive treatments are emerging to mitigate the risk of metastases following surgery, the need for effective prognostic tools in the organ confined disease state to estimate risk is growing exponentially. Several ongoing and completed trials have focused on vascular endothelial growth factor receptor (VEGFR) tyrosine kinases inhibitors (TKIs) in the adjuvant setting [1-3], a large study recently completed evaluating mTOR inhibition in this setting [4], and newer studies are now testing immunotherapy as a surgical adjunct for mitigation of risk $[5,6]$. Challenges with these agents and approaches are the uncertainty of risk, in addition to the uncertainty as to how effectively non-cytotoxic drugs can clear micrometastatic disease.

It is perhaps not surprising that the early data from these studies have been challenging to interpret. Data from clinical trials ASSURE, S-TRAC and PROTECT using sunitinib/sorafenib, sunitinib and pazopanib, respectively, have shown conflicting results, leading to confusion and diverse opinions regarding the application of these treatments. The first study to report a positive outcome was the STRAC study, showing a disease-free survival (DFS) benefit of 1.2 years (HR 0.76 ; $95 \%$ CI, 0.59 to 0.98 ; $P=0.03$ ) [1]. The overall survival (OS) data of the S-TRAC trial are not yet mature. In the most updated analysis, median OS was not reached in either arm (HR 0.92, 95\% CI 0.66-1.28; $p=0.6$ ) but the benefit of sunitinib over placebo was observed across all subgroups [7]. Regardless of whether the rate for cure is improved, there is substantial enthusiasm around the delay in recurrence. The ASSURE trial randomized patients to sunitinib vs sorafenib vs placebo and found no difference in DFS, the primary endpoint of the study (median DFS was 5.8 years for sunitinib [HR 1.02; 97.5\% CI: 0.85-1.23; $P=0.8038$ ], 6.1 years for sorafenib (HR: 0.97; 97.5\% CI: 0.80-1.17; $P=0.7184$ ), and 6.6 years for placebo) [2]. Similarly the PROTECT trial, randomizing patients to pazopanib vs placebo, also did not meet the primary DFS endpoint in the ITT $600 \mathrm{mg}$ dose (HR: $0.862 ; 95 \%$ CI, 0.699, 1.063; $p=0.165)$. The secondary analysis of DFS in ITT $800 \mathrm{mg}(n=403)$ yielded an HR of 0.69 (95\% CI, 0.51 to 0.94) [8]. Nonetheless, systemic therapy for risk mitigation in the post-nephrectomy setting is fast becoming a clinical reality. Based on the S-TRAC data, in November 2017, the FDA approved adjuvant sunitinib in localized high risk RCC following nephrectomy. However, given the negative results of ASSURE and conflicting results of the PROTECT trial, physicians need to evaluate the risk-benefit ratio, discuss the results with patients and make a joint decision before initiating adjuvant sunitinib.

Established clinical risk prediction algorithms and nomograms such as the Stage Size Grade and Necrosis (SSIGN) score [9] and the UCLA Integrated Staging System [10] were used in these adjuvant clinical trials to risk-stratify patients. Unfortunately, sub-analysis of ASSURE patients including the same high-risk criteria as in the S-TRAC trial, did not show a similar DFS benefit. Although the value of accurate prognostic stratification has not been demonstrated in selecting or electing to apply adjunctive therapy in ccRCC, the patient selection for adjuvant therapy will soon present a major dilemma in this venue. This challenge further underscores the need for objective molecular criteria for robust application of molecular based prognostic markers in the context of clinical prognostic criteria could help better predict groups of patients who will most benefit from such adjuvant treatment approaches.

This review will evaluate the molecular signature ClearCode 34 , discuss its validation, and consider the rational application of molecular prognostic risk assessment tools as a strategy to guide future applications of adjunctive therapy in ccRCC.

\section{MOLECULAR SUB-TYPING RCC}

Several molecular and gene expression profiles have been published in the last decade to help classify 
tumors based on their clinical aggressiveness. In 2015, Rini et al. developed a 16-gene recurrence score to help predict recurrence in patients with stage I-III ccRCC [11]. Expression of 732 genes was measured by reverse-transcription PCR and clinical outcome in 942 patients with stage I-III ccRCC who had undergone nephrectomy was studied, and 11 genes combined with 5 reference genes (16 genes in total) were associated with recurrence. This score has also been rigorously validated in an external cohort. However, this score has not yet entered clinical application.

Certainly, the major subtypes of renal cell carcinomas have distinct behaviors, but previously lacked molecular definitions. Through the large-scale efforts of The Cancer Genome Atlas (TCGA) and other groups, molecular profiles clearly defining the three major subtypes of RCC-ccRCC, papillary renal cell carcinoma and chromophobe renal cell carcinoma- were identified [12-14]. Buttner et al. recently developed the S-3 score based on expression data of ccRCC, papillary and chromophobe RCC from the TCGA database, and correlated it with cancer-specific survival [15]. As traditional histologic strategies and molecular profiles are merging in the pathological diagnosis of cancers, the potential for more robust prognostic stratification in many cancers is a realistic expectation.

\section{TRANSCRIPT PROFILING FOR ccRCC PROGNOSIS}

Prior to the discovery of these commonly mutated genes in ccRCC via next generation sequencing efforts, transcript-based studies were investigated to find signals in the gene expression outputs of ccRCC tumors that could lend insight into the features that relate to the variable natural history that has long been a hallmark of this disease. The initial analysis used an unbiased approach to identify naturally occurring patterns in the transcript profile, independent of any outcome-based modeling. This strategy is thus geared to identifying biologically meaningful and inherently more stable features for classifying subsets of disease. The initial project elected to focus on clear cell tumors, recognizing that the other histology RCC tumors likely represented distinct molecular entities.

Fifty-one specimens from $48 \mathrm{ccRCC}$ patients were collected from patients undergoing nephrectomy for RCC at the Vanderbilt University from 1994 to 2008. Brannon et al. used genome-wide messenger RNA microarray using the Agilent $44 \mathrm{~K}$ platform to define gene transcription across genome wide expression. Data of approximately 5000 genes, representing the greatest variability in expression was used to model dominant molecular subtypes of ccRCC tumors, using consensus clustering methods. Ultimately the most stable model implied two dominant subgroups, termed clear cell A (ccA) and clear cell B (ccB) based on unsupervised clustering $[16,17]$. Based on the core tumors, or most well-defined arrays, in each subtype, logical analysis of data (LAD) defined a small, highly predictive gene set that could then be used to classify additional tumors individually. The subclasses were corroborated in a validation data set of 177 tumors and analyzed for clinical outcome, where the association with prognosis was established.

Biologically, these subtypes were highly distinct: ccA tumors exhibit increased angiogenesis and signature hypoxic signaling, corresponding to the classical features associated with ccRCC. While ccB tumors displayed lower hypoxia gene expression, these tumors also demonstrated a number of interesting features associated with aggressive cancer growth, including increased transforming growth factor $b$ (TGF-b) and epithelial-to-mesenchymal signaling. These findings were particularly compelling that the features drove a biological classification, which also tied to poor outcome measures. The ccB classified tumors demonstrated increased tumor size, grade, and rate of metastasis as well as decreased recurrencefree survival (RFS), cancer-specific survival (CSS) and $\mathrm{OS}$ in the original, as well as other multiple data sets $[17,18]$.

\section{APPLICATION TO CLINICAL TISSUES}

The initial studies utilized fresh frozen tissue and large number of gene probes, leading to significant barriers to translational and clinical applications. Brooks et al. further developed a model using 34 genes (ClearCode34) that classified tumors as ccA and $\mathrm{ccB}$ from FFPE specimens [18]. These genes were selected for having large changes in gene expression corresponding to each subtype, increasing the robustness of profile identification. NanoString technology was used to measure expression profiles in 72 ccRCC samples, obtained from an FFPE archive. These tumors were chosen as references to develop the 34-gene panel based on concordant subtype classifications determined by two methods: logical analysis of data and Consensus Cluster. Tumor 
classification was performed in $\mathrm{R}$ program using Prediction Analysis of Microarrays for R (PAMR), a centroid-based classification algorithm.

These tumors were also selected to represent primary tumors with significant clinical risk for recurrence, primarily stage 2 and 3 , and excluding early stage tumors. These intermediate stage tumors were considered to represent the critical group, for which a prognostic tool would be of greatest value. Since stage 1 tumors have such a good clinical outcome, little value is added in a molecular phenotype that informs a low risk for recurrence. However, the exclusion of this group of patients challenges the interpretation of this score if it is applied in this tumor setting. On the other end of the spectrum, stage 4 tumors carry enormous risk, and as metastatic disease portends a different natural history, these tumors are also not in need of robust molecular classification regarding recurrent risk. Stage 2 and 3 , however, encompasses a wide range of risk for disease recurrence, and subjective histologic assessments regarding grade (which is subtly different between grading features for grade 2 and grade 3 ). Of the 34 genes, 10 genes were derived from $\mathrm{ccB}$ cohort and the remaining 24 genes were derived from the ccA cohort (Table 1).

\section{INTEGRATION WITH CLINICAL RISK ASSESSMENT}

The Brooks et al. paper further performed an integrated scoring strategy to include clinical risk assessment with molecular phenotyping. The ClearCode34 assignment was independently correlated with outcome in multivariate analysis that included stage, grade, tumor size, presence of necrosis, and performance status. As an integrated tool, the risk assessment outperformed both UISS and S-SIGN in assessment of risk for disease recurrence [18].

Another unvalidated feature that has been long considered to be a hallmark of more aggressive disease is the presence of Fluoro-deoxyglucose (FDG) uptake in tumor cells. This feature, measured by conventional PET imaging, is a challenge for monitoring ccRCC, owing to the abundant tumors showing no evidence of F-18 labeled FDG uptake, which can be confounding when this radiographic tool is used for disease detection or monitoring. Using high resolution imaging MR-PET, samples of tumors that were classified as FDG avid (standard uptake value $>2$ ) were consistently represented in the ccB cohort,
Table 1

ClearCode34 genes

\begin{tabular}{|c|c|}
\hline Genes & Classification \\
\hline MAPT & $\mathrm{ccA}$ \\
\hline STK32B & $\operatorname{ccA}$ \\
\hline FZD1 & $\operatorname{ccA}$ \\
\hline RGS5 & $\mathrm{ccA}$ \\
\hline GIPC2 & $\operatorname{ccA}$ \\
\hline PDGFD & $\mathrm{ccA}$ \\
\hline EPAS1 & $\operatorname{ccA}$ \\
\hline MAOB & $\operatorname{ccA}$ \\
\hline $\mathrm{CDH} 5$ & $\mathrm{ccA}$ \\
\hline TCEA3 & $\mathrm{ccA}$ \\
\hline LEPROTL1 & $\operatorname{ccA}$ \\
\hline BNIP3L & $\mathrm{ccA}$ \\
\hline EHBP1 & $\mathrm{ccA}$ \\
\hline VCAM1 & $\operatorname{ccA}$ \\
\hline PHYH & $\mathrm{ccA}$ \\
\hline PRKAA2 & $\mathrm{ccA}$ \\
\hline SLC4A4 & $\mathrm{ccA}$ \\
\hline ESD & $\mathrm{ccA}$ \\
\hline TLR3 & $\mathrm{ccA}$ \\
\hline NRP1 & $\mathrm{ccA}$ \\
\hline C11orf11 & $\mathrm{ccA}$ \\
\hline ST13 & $\mathrm{ccA}$ \\
\hline ARNT & $\mathrm{ccA}$ \\
\hline C13orf1 & $\mathrm{ccA}$ \\
\hline SERPINA3 & $\mathrm{ccB}$ \\
\hline SLC4A3 & $\mathrm{ccB}$ \\
\hline MOXD1 & $\mathrm{ccB}$ \\
\hline KCNN4 & $\mathrm{ccB}$ \\
\hline ROR2 & $\mathrm{ccB}$ \\
\hline FLJ23867 & $\mathrm{ccB}$ \\
\hline FOXM1 & $\mathrm{ccB}$ \\
\hline UNG2 & $\mathrm{ccB}$ \\
\hline GALNT10 & $\mathrm{ccB}$ \\
\hline GALNT4 & $\mathrm{ccB}$ \\
\hline
\end{tabular}

whereas, FDG nonavid tumors corresponded with the ccA classification [19].

\section{ASSOCIATION WITH COMORBIDITY}

Seeking to understand the host features that correspond with disease prognosis or behavior, Haake et all determined the association of ccA or ccB status with comorbidities at the time of diagnosis. Intriguingly, since in many other malignancies obesity is tied to worse outcome, ccA patients were more likely to be obese ( $48 \%$ vs $34 \%, p=0.021)$ and diabetic ( $26 \%$ vs $13 \%, p=0.035$ ) [20]. In multivariable analysis, diabetes was associated with inferior CSS and OS despite being associated with the superior prognostic group, ccA. Given the increased incidence of obesity and diabetes in ccA patients, the question of whether host clinical features can impact molecular subtypes and tumor biology is raised. Future prospective studies are needed to validate the question further. 
Table 2

Reported survival outcomes in published studies validating ClearCode34 in metastatic and non-metastatic RCC patients

\begin{tabular}{|c|c|c|c|c|c|c|}
\hline & $N$ & Source & Stage & $\begin{array}{c}\text { RFS } \\
\text { ccA vs ccB }\end{array}$ & $\begin{array}{c}\text { OS } \\
\text { ccA vs ccB }\end{array}$ & $\begin{array}{c}\text { CSS } \\
\text { ccA vs ccB }\end{array}$ \\
\hline Brooks et al. [16] & $157(69 \mathrm{ccA}, 88 \mathrm{ccB})$ & UNC (FFPE, NanoString) & Non-metastatic & $\mathrm{HR}=12.2$ & $\mathrm{HR}=5.52$ & $\mathrm{HR}=12.45$ \\
\hline Haake et al. [18] & $282(226 \mathrm{ccA}, 56 \mathrm{ccB})$ & Moffitt (fresh frozen) & Non-metastatic & - & $\mathrm{HR}=2.50$ & $\mathrm{HR}=3.26$ \\
\hline De Velasco et al. [19] & $54(17 \mathrm{ccA}, 37 \mathrm{ccB})$ & $\begin{array}{l}\text { Dana-Farber, MDACC, U } \\
\text { Pittsburgh, MSKCC, UNC }\end{array}$ & Metastatic & - & $\mathrm{HR}=2.33$ & - \\
\hline Serie et al. [22] & $91(47 \mathrm{ccA}, 44 \mathrm{ccB})$ & TCGA & Metastatic & - & $\mathrm{HR}=1.73$ & $\mathrm{HR}=1.70$ \\
\hline $\begin{array}{l}\text { Eckel-Passow et al. } \\
\text { [23] }\end{array}$ & $417(220 \mathrm{ccA}, 197 \mathrm{ccB})$ & TCGA & $\begin{array}{l}\text { Metastatic and } \\
\text { Non-metastatic }\end{array}$ & - & $\mathrm{HR}=1.88$ & - \\
\hline
\end{tabular}

*NA, Not assessed.

The ccA patients also trended towards having more frequent use of angiotensin system inhibitors (71\% vs $52 \%, p=0.055)$. Similar rates of hyperlipidemia and statin utilization were observed in the two subtypes. Additionally, smoking rates were similar in each subtype.

\section{VALIDATION TESTING}

Both $\mathrm{ccA} / \mathrm{ccB}$ and ClearCode 34 have been validated in several independent datasets. Brooks et al. used the TCGA cohort to allow testing of the prognostic value of ClearCode34 in an independent data set, revealing that $\mathrm{ccA}$ and $\mathrm{ccB}$ were significantly associated with multiple survival end points, which was also observed in the UNC cohort derived from FFPE tissues [18] (Table 2).

In a retrospective analysis of 282 patient samples from Moffitt Cancer Center with ccRCC, analyzed for gene expression based on their institutional algorithm, ClearCode34 was applied to identify tumors as $\mathrm{ccA}$ and $\mathrm{ccB}$. The superior OS, CSS and RFS was validated in ccA patients relative to the $\mathrm{ccB}$ patients $(p<0.001)$ [20]. Univariate analysis demonstrated that ccA patients, relative to $\mathrm{ccB}$ patients, presented with lower Fuhrman grade $(p<0.001)$ and smaller tumors $(p=0.003)$ and were less likely to have nodal involvement ( $p=0.038$ ) or metastatic disease ( $p=0.003)$. In multivariable analysis, $c c B$ status was independently associated with inferior CSS (HR $3.26,95 \%$ CI 1.84-5.79) and OS (HR 2.5, 95\% CI 1.53-4.08) (Table 2).

De Velasco et al. recently validated ClearCode 34 in patients with metastatic ccRCC. ClearCode 34 stratified 54 patients with metastatic ccRCC into two groups $-\operatorname{ccA}(n=17)$ and ccB $(n=37)$. Patients in the ccB group had significantly worse OS (HR: $2.33,95 \% \mathrm{CI}, 1.02-5.31 ; p=0.039)$. In multivariable analysis, adjusting for MSKCC or IMDC criteria, $\mathrm{ccB}$ remained independently associated with a worse OS [21].

Similarly, Serie et al. identified 111 patients who had tissue available from their primary RCC tumor and at least one metastasis. ClearCode34 genes were analyzed for all tumors. Patients classified to ccB subtype had significantly worse cancer specific survival (age- and gender-adjusted HR: 1.70, 95\% confidence interval [CI] 1.07-2.71, $p=0.025$ ) and overall survival (age- and gender-adjusted HR 1.73, 95\% CI $1.10-2.73, p=0.018$ ) in comparison to ccA [22].

Eckel-Passow et al. used the TCGA database and assigned patients to the $\mathrm{ccA} / \mathrm{ccB}$ subtypes, finding significant association with tumor size, grade and necrosis [23]. Although the association of the $\mathrm{ccA} / \mathrm{ccB}$ molecular subtypes with overall survival is attenuated after adjustment for age and the Mayo SSIGN score, the ccA/ccB subtypes remained statistically significant. Specifically, after adjusting for both age and the SSIGN score, patients in the ccB subtype had a significantly worse survival in comparison with patients in the ccA subtype $(\mathrm{HR}=1.88$, $P=0.003$ ) [23].

In the development of ClearCode34, the risk assessment tool was also compared with existing clinical nomograms to predict death from ccRCC, using multivariate and co-occurrence index (C-index) analysis. This analysis showed superiority in assessing risk of ccRCC death by C-index analysis using the ClearCode34 model compared to the UISS/SSIGN score [18]. However, it should be noted that in this cohort, the C-index for UISS/SSIGN score was much lower compared to the earlier published $\mathrm{C}$-indices for UISS/SSIGN [24] (C-statistic 0.62 vs 0.83 ). This may reflect differences in patient populations, especially higher risk patients represented in the ClearCode 34 study cohort, and the exclusion of early stage patients as noted above. 


\section{EFFECTS OF HETEROGENEITY}

One issue that has substantially confounded the field of tumor-specific biomarker development in ccRCC is intratumoral heterogeneity. Originally demonstrated in a landmark study that subsampled a large primary tumor and associated metastasis, ccRCC tumors have emerged as being a representative tumor for demonstrating branched tumor evolution from a small number of core (or 'truncal') mutations [25]. The expression-based classifiers, ccA and $\mathrm{ccB}$ were evaluated in the original publication and the majority of samples classified as ccB subgroup in the primary tumor. However, one sample classified as ccA, even upon repeat analysis using ClearCode34 algorithms (unpublished data). Thus, prognostic gene expression signatures may not correctly predict outcomes if they are assessed from a single region of a heterogeneous tumor. Due to intratumor heterogeneity, individual tumors can harbor both ccA and ccB components, and while this may provide insight as to the developmental stage of this tumor, this property renders a single sample classification strategy difficult for informing therapeutic selection. Gulati et al. evaluated multiple biopsy samples from each tumor and observed that 8 of 10 $(80 \%)$ patients had a primary tumor that exhibited both ccA and ccB subtypes. Such effects of intratumoral heterogeneity may be possible to overcome if strict pathologic review is conducted and the section with the most aggressive tumor characteristics (e.g. grade) is selected for molecular analysis [22, 26]. Alternatively, since the degree of ccB present in the tumor is ultimately the feature that is most desired for making a prognostic assessment, the correlation with FDG PET avidity may provide an opportunity for radiographic prognosis to be incorporated into the disease algorithm, and ultimately to lend insight into the disease heterogeneity.

\section{CONCLUSIONS}

Several molecular profiles have been developed to predict prognosis of patients with localized RCC, but these tools have not yet been applied in direct comparisons. ClearCode 34 is a highly validated profile that can aid prognosis based on molecular features of the primary tumor. Several caveats have been developed, including the limited application in small renal masses, confounding issues around tumor heterogeneity, and the potential for misclassification based on sample bias. In spite of these known limitations, this tool has been consistent in prognostic assessment. Strategies to overcome the issues around tumor heterogeneity should only make this marker more robust in applying a prognostic classification scheme for patients with intermediate risk tumors post nephrectomy. It is also worth note, that the same level of heterogeneity is detectable in a number of tumors in addition to ccRCC, where expression-based biomarkers continue to play a major role in disease assessment. Additionally, the experience with ClearCode34 in metastatic lesions is too limited at this time to determine if a role exists outside the prognosis of risk for recurrence.

The application of a prognostic risk assessment tool for patients with localized disease is most valuable if it can guide an intervention that alters risk. Presently, prognostic tools have application that is limited to defining strategies for disease surveillance. Adjuvant therapy for ccRCC remains in it's infancy, although the first FDA approval of a therapeutic in this setting increases the urgency for developing strategies around patient selection. Ongoing trials will be expected to investigate these issues vigorously. Molecular classification may provide an opportunity to classify patients who could benefit from adjuvant therapeutic options based on risk for recurrence, but needs to be tested prospectively, and compared with other emerging biomarkers. While there is a demonstrated prognostic role of ClearCode34, there is a need to incorporate molecular profiles in prospective clinical trials to help determine the best use of molecular information in guiding clinical decisions.

\section{CONFLICT OF INTEREST}

The authors declare no conflict of interest.

\section{ACKNOWLEDGMENTS}

This collaboration reflects activity on NIH K24 CA172355 (WKR) and was made possible due to the connections made at the AACR Molecular Biology in Clinical Oncology Workshop.

\section{REFERENCES}

[1] Ravaud A, et al. Adjuvant sunitinib in high-risk renalcell carcinoma after nephrectomy. N Engl J Med 2016; 375(23):2246-54.

[2] Haas NB, et al. Adjuvant sunitinib or sorafenib for highrisk, non-metastatic renal-cell carcinoma (ECOG-ACRIN 
E2805): A double-blind, placebo-controlled, randomised, phase 3 trial. Lancet 2016;387(10032):2008-16.

[3] Sternberg CN, et al. Pazopanib in locally advanced or metastatic renal cell carcinoma: Results of a randomized phase III trial. J Clin Oncol 2010;28(6):1061-8.

[4] NCT01120249, Everolimus in treating patients with kidney cancer who have undergone surgery. Available at: http://www.clinicaltrials.gov. [last accessed 10/26/17].

[5] Harshman L. A phase III randomized study comparing perioperative nivolumab vs. observation in patients with localized renal cell carcinoma undergoing nephrectomy (PROSPER RCC). Journal of Clinical Oncology 35(15_suppl).

[6] NCT03024996, A Study of Atezolizumab as Adjuvant Therapy in Participants With Renal Cell Carcinoma (RCC) at High Risk of Developing Metastasis Following Nephrectomy (IMmotion010) Available at: http://www. clinicaltrials.gov [last accessed 10/26/17].

[7] Motzer RJ, et al. Adjuvant Sunitinib for High-risk Renal Cell Carcinoma After Nephrectomy: Subgroup Analyses and Updated Overall Survival Results. Eur Urol 2017.

[8] Motzer R. Randomized phase III trial of adjuvant pazopanib versus placebo after nephrectomy in patients with locally advanced renal cell carcinoma (RCC) (PROTECT). Journal of Clinical Oncology 2017;35(15_suppl):4507-7.

[9] Frank I, et al. An outcome prediction model for patients with clear cell renal cell carcinoma treated with radical nephrectomy based on tumor stage, size, grade and necrosis: The SSIGN score. J Urol 2002;168(6):2395-400.

[10] Lam JS, et al. Postoperative surveillance protocol for patients with localized and locally advanced renal cell carcinoma based on a validated prognostic nomogram and risk group stratification system. J Urol 2005;174(2):466-72; discussion 472; quiz 801.

[11] Rini B, et al. A 16-gene assay to predict recurrence after surgery in localised renal cell carcinoma: Development and validation studies. Lancet Oncol 2015;16(6):676-85.

[12] Network CGAR. Comprehensive molecular characterization of clear cell renal cell carcinoma. Nature 2013;499(7456):43-9.

[13] Linehan WM, et al. Comprehensive molecular characterization of papillary renal-cell carcinoma. $\mathrm{N}$ Engl J Med 2016;374(2):135-45.

[14] Davis CF, et al. The somatic genomic landscape of chromophobe renal cell carcinoma. Cancer Cell 2014;26(3):319-30.
[15] Büttner F, et al. Survival prediction of clear cell renal cell carcinoma based on gene expression similarity to the proximal tubule of the nephron. Eur Urol 2015;68(6):1016-20.

[16] Brannon AR, et al. Molecular stratification of clear cell renal cell carcinoma by consensus clustering reveals distinct subtypes and survival patterns. Genes Cancer 2010;1(2):152-63.

[17] Brannon AR, et al. Meta-analysis of clear cell renal cell carcinoma gene expression defines a variant subgroup and identifies gender influences on tumor biology. Eur Urol 2012;61(2):258-68.

[18] Brooks SA, et al. ClearCode34: A prognostic risk predictor for localized clear cell renal cell carcinoma. Eur Urol 2014;66(1):77-84.

[19] Brooks SA, et al. Alternate metabolic programs define regional variation of relevant biological features in renal cell carcinoma progression. Clin Cancer Res 2016;22(12): 2950-9.

[20] Haake SM, et al. Patients with ClearCode34-identified molecular subtypes of clear cell renal cell carcinoma represent unique populations with distinct comorbidities. Urol Oncol 2016;34(3):122.e1-7.

[21] de Velasco G, et al. Molecular subtypes improve prognostic value of international metastatic renal cell carcinoma database consortium prognostic model. Oncologist 2017;22(3):286-92.

[22] Serie DJ, et al. Clear cell type A and B molecular subtypes in metastatic clear cell renal cell carcinoma: Tumor heterogeneity and aggressiveness. Eur Urol 2017;71(6):979-85.

[23] Eckel-Passow JE, et al. Assessing the clinical use of clear cell renal cell carcinoma molecular subtypes identified by RNA expression analysis. Urol Oncol 2015;33(2):68. e17-23.

[24] Zigeuner R, et al. External validation of the Mayo Clinic stage, size, grade, and necrosis (SSIGN) score for clear-cell renal cell carcinoma in a single European centre applying routine pathology. Eur Urol 2010;57(1):102-9.

[25] Gerlinger M, et al. Intratumor heterogeneity and branched evolution revealed by multiregion sequencing. $\mathrm{N}$ Engl J Med 2012;366(10):883-92.

[26] Callea M, et al. Differential expression of PD-L1 between primary and metastatic sites in clear-cell renal cell carcinoma. Cancer Immunol Res 2015;3(10):1158-64. 